\title{
Los distritos rurales: un nuevo concepto de desarrollo territorial. Modelos Centro- Periferia en Castilla-La Mancha
}

\author{
JUAN SEBASTIÁN CASTILLO VALERO \\ Instituto de Desarrollo Regional. UNIVERSIDAD DE CASTILLA-LA MANCHA, ESPAÑA. \\ E-mail: sebastian.castillo@uclm.es
}

Ma CARMEN GARCÍA CORTIJO

Instituto de Desarrollo Regional. UNIVERSIDAD DE CASTILLA-LA MANCHA, ESPAÑA. E-mail: mariacarmen.gcortijo@uclm.es

\begin{abstract}
RESUMEN
El movimiento demográfico y la desconcentración en las áreas urbanas y sus áreas periféricas, conforman, en los últimos treinta años, un proceso distinto de distribución espacial y la definición de un nuevo modelo de asentamiento de la población. Tradicionalmente, eran desplazamientos rural-urbano y la principal razón era la económica. Sin embargo, el proceso más reciente es más complejo y más triangular: movimientos rural-rural y corredores urbanos, el movimiento de la población se realiza de los núcleos más pequeños (periferia) a la cabecera comarcal (centro), la dimensión económica no logra explicar la mayor parte de los desplazamientos, sino que aparecen los núcleos rurales más grandes como concentradores de la población absorbida de los núcleos periféricos, conformando un distrito social frente al tradicional distrito industrial de tradición marshalliana, y que nosotros denominamos como Distrito Rural que se convierte, así, en un nuevo concepto para el desarrollo territorial.
\end{abstract}

Palabras clave: distrito rural, economías de escala sociales, modelos hedónicos poblacionales, centro-periferia.

\section{Rural Districts: a New Concept of Territorial Development. Centre-Outskirts Models in Castilla-La Mancha}

\begin{abstract}
The population movement and the decentralization of urban areas and their outskirts are shaping, in the last thirty years, a different process of spatial distribution and the definition of a new population establishment model. Traditionally, displacements were rural-urban and the major reason was the economic one. Nevertheless, recently this process is more complex and triangular: rural-rural displacements and intern urban migrations in which the population moves from small cores (outskirts) to the capital (urban centre). The economic angle is not able anymore to explain most of the displacements: the biggest rural cores are concentrating the migration flows of outskirt areas, shaping, instead of a traditional marshallian industrial district, a social district that we have named Rural District, which becomes a new concept for territorial development.
\end{abstract}

Keywords: rural district, social economies of scale, hedonic population models, centre-outskirts.

Clasificación JEL: C01, J11, R00

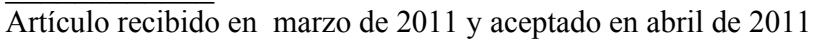

Artículo disponible en versión electrónica en la página www.revista-eea.net, ref. ə-29113 


\section{INTRODUCCIÓN}

\subsection{Tendencias sociodemográficas del medio rural y urbano}

Como señala Camarero (1993), a finales del siglo XX el secular proceso de despoblamiento rural parece tocar fondo en los países avanzados. Las primeras evidencias se observan hacia los años 1970, en EEUU, donde el crecimiento demográfico era mayor en las áreas no metropolitanas que en las metropolitanas, manifestándose, por tanto, una inflexión en la tendencia del crecimiento urbano. Por primera vez, los núcleos centrales dejaron de atraer efectivos poblacionales e iniciaron un lento declive en el número de sus habitantes mientras que sus periferias residenciales continuaban creciendo. A la vez, otras áreas rurales distantes iniciaron un substancial crecimiento demográfico basado, esencialmente, en los desplazamientos definitivos de población.

Dichas variaciones, como indica Arroyo (2001), parecían no obedecer a las mismas condiciones en las que se habían desarrollado, hasta entonces, los modelos clásicos de urbanización e hicieron suponer que se había producido un cambio en las tendencias de los movimientos migratorios urbanos. Inicialmente, se creyó que la crisis de los años setenta estaba incidiendo sobre la estructura económica del sistema y que ésta era la causa de que los centros metropolitanos perdiesen población; pero, también, se observó que otros centros urbanos menores no parecían sentirse afectados por dicha situación. Además, el hecho de que ese fenómeno se hubiese observado en países fuertemente industrializados hizo pensar que el grado de industrialización y los nuevos movimientos migratorios podían guardar relación.

Este mismo proceso, también, se confirmó en Japón, Suiza, Noruega, Italia, Dinamarca, Nueva Zelanda, Bélgica, Francia, Alemania y Holanda que fueron denominados los "turnaround countries". España no fue indiferente a estos cambios, aunque fue un fenómeno más tardío. España en los años sesenta estaba en pleno apogeo industrial-urbano, mientras en el resto ya se conocían procesos de descentralización de la industria y de la población. Este aspecto derivó, como señala Ferrás (2007), que estudios acerca de este tema fueran también tardíos. El antecedente más antiguo se retrotrae a Allende (1983) que presentó el declive urbano en los Estados Unidos y Gran Bretaña como una experiencia que se podría desarrollar en España. A partir de 1985, destacan las investigaciones empíricas de Bernabé y Albertos (1986), Ogando, Pedrosa y Fernández (1989), Belil (1990) y Benito (1991), entre otros.

En definitiva, en los países desarrollados, desde 1970, y en España, a partir de 1980, se empezó a experimentar un nuevo proceso poblacional: las zonas urbanas perdieron peso y la jerarquía urbana y las relaciones centro-periferia cambiaron hacia unas relaciones menos jerárquicas y multipolares. 
En el caso de mundo rural, los movimientos se ralentizaron, pero el signo de caída demográfica fue inexorable, y sólo ha sido, en las dos últimas décadas, cuando ha surgido un cambio cualitativo: el movimiento rural-rural, de los núcleos de menor dimensión a las cabeceras comarcales y/o núcleos de mayor población, en una reproducción de un modelo centro-periferia en el nivel territorial rural. Las razones y fuerzas motrices de este cambio están vinculadas a la aparición y reproducción de mecanismos insertos dentro de la nueva geografía económica de Krugman (1991) pero sobre bases sociales en lugar de económicas, lo que le confiere un verdadero rasgo característico y novedoso y sobre todo, conceptualmente, una dimensión teórica atractiva: la conformación de los distritos rurales, entendidos como una evolución especifica del concepto de distrito industrial de origen marshalliano, con una base social, en el caso del distrito rural, en las economías de escala centrípetas en lugar de la base productiva presente en los distritos industriales.

\subsection{Contexto sociodemográfico de Castilla-La Mancha}

Dentro del marco anteriormente descrito, el cambio cualitativo en las pautas de movilidad migratoria, de ruptura del modelo unidireccional de urbanización y desruralización y génesis de una situación nueva de intercambio poblacional entre zonas rurales y urbanas que han afectado a España, parece pertinente analizar los movimientos migratorios desde un nivel inferior: las regiones. En este sentido, nosotros nos centramos en el caso de Castilla-La Mancha, sobre el que no existe ningún estudio al respecto, además de ser una de las escasas comunidades autónomas en la que la población rural sobrepasa el $50 \%$ de la población total.

Para ello es necesario poner en contexto la tendencia demográfica del medio rural y urbano en Castilla-La Mancha. Así, siguiendo la clasificación del Instituto Nacional de Estadística que define a las zonas rurales como aquellas entidades con menos de 2.000 habitantes y a las zonas urbanas como entidades de más de 10.000 habitantes; compararemos la evolución poblacional de ambos ámbitos con diferentes variables (asimilando en determinados territorios rurales, los centros comarcales como aquellos núcleos de mayor población presentes, cuando no se llega al limite establecido de los 10.000 habitantes). En la Tabla 1, calculamos las tasas de variación de la población, desde 1950 hasta la actualidad. 
Tabla 1

Tasas de Variación (\%) de la Población de los municipios < 2.000 habitantes

\begin{tabular}{|c|c|c|c|c|c|c|}
\hline RURAL & $\mathbf{1 9 5 0 - 6 0}$ & $\mathbf{1 9 6 0 - 7 0}$ & $\mathbf{1 9 7 0 - 8 0}$ & $\mathbf{1 9 8 0 - 9 0}$ & $\mathbf{1 9 9 0 - 2 0 0 0}$ & $\mathbf{2 0 0 0 - 2 0 1 0}$ \\
\hline Albacete & $-12,02$ & $-28,66$ & $-22,74$ & $-7,14$ & $-15,53$ & $-7,09$ \\
\hline Ciudad Real & $-0,83$ & $-31,00$ & $-25,43$ & $-1,42$ & 45,29 & $-36,02$ \\
\hline Cuenca & $-10,24$ & $-28,53$ & $-24,56$ & $-3,67$ & $-13,45$ & $-6,06$ \\
\hline Guadalajara & $-13,20$ & $-30,96$ & $-33,28$ & $-4,23$ & $-9,62$ & 19,33 \\
\hline Toledo & $-7,83$ & $-28,45$ & $-21,41$ & 1,94 & $-6,72$ & 13,40 \\
\hline
\end{tabular}

Fuente. Elaboración propia con datos INE.

Independientemente de que el signo de las tasas sea positivo o negativo, sí que puede hablarse, en cierto sentido, de la existencia de un punto de inflexión en los procesos de concentración de la población a partir de 1980. Si bien, Castilla-La Mancha, de 1960 a 1980, sufre un declive de la población rural, debido a la mejora de las expectativas de empleo en las urbanas, es a partir de 1980 cuando se rompe esta tendencia, aún a pesar de la disminución de la población pero, no de forma tan acentuada; manifestándose incluso signos de recuperación demográfica en las provincias de Guadalajara y Toledo en la década actual.

Sin embargo, como señala Camarero (1993), para evidenciar esa recuperación demográfica en las zonas rurales es necesario comprobar que la reducción de las distancias entre el medio rural y el urbano también se ha acortado; para ello, calculamos las diferencias entre las tasas de variación calculadas para los municipios de menos de 2.000 habitantes (Tabla 1) y las de los municipios de más de 10.000 habitantes, obteniendo, así, la distancia real entre ambos medios (Tabla 2). Nuevamente, los datos, tanto negativos como positivos de las tasas de variación, nos indican que, desde 1980, se acortan las diferencias entre ambas porque la cuantía de las tasas negativas va en disminución y la de las positivas en aumento (Tabla 2).

Tabla 2

Diferencias entre las Tasas de Variación (\%) de la Población de los municipios $>10.000$ habitantes y de la Población de los municipios < 2000 habitantes

\begin{tabular}{|c|c|c|c|c|c|c|}
\hline $\begin{array}{c}\text { Distancia Medio } \\
\text { Rural y Urbano }\end{array}$ & $\mathbf{1 9 5 0 - 6 0}$ & $\mathbf{1 9 6 0 - 7 0}$ & $\mathbf{1 9 7 0 - 8 0}$ & $\mathbf{1 9 8 0 - 9 0}$ & $\mathbf{1 9 9 0 - 2 0 0 0}$ & $\mathbf{2 0 0 0 - 2 0 1 0}$ \\
\hline Albacete & $-37,66$ & $-54,32$ & $-35,39$ & $-15,23$ & $-18,04$ & $-16,93$ \\
\hline Ciudad Real & $-27,01$ & $-34,4$ & $-20,45$ & $-71,86$ & $-38,31$ & 43,67 \\
\hline Cuenca & 16,07 & 67,52 & 84,75 & 7,09 & 57,18 & 215,9 \\
\hline
\end{tabular}




\section{Tabla 2 (continuación)}

Diferencias entre las Tasas de Variación (\%) de la Población de los municipios $>10.000$ habitantes y de la Población de los municipios < 2000 habitantes

\begin{tabular}{|c|c|c|c|c|c|c|}
\hline $\begin{array}{c}\text { Distancia Medio } \\
\text { Rural y Urbano }\end{array}$ & $\mathbf{1 9 5 0 - 6 0}$ & $\mathbf{1 9 6 0 - 7 0}$ & $\mathbf{1 9 7 0 - 8 0}$ & $\mathbf{1 9 8 0 - 9 0}$ & $\mathbf{1 9 9 0 - 2 0 0 0}$ & $\mathbf{2 0 0 0 - 2 0 1 0}$ \\
\hline Guadalajara & 22,82 & 136,19 & $-292,13$ & $-35,79$ & $-37,2$ & $-39,94$ \\
\hline Toledo & $-237,29$ & $-86,33$ & $-51,62$ & 41,89 & $-40,46$ & $-23,95$ \\
\hline
\end{tabular}

Fuente. Elaboración propia con datos INE.

Otra variable que muestra que las tendencias demográficas están cambiando y los pueblos están empezando a recobrar vitalidad demográfica es la de las variaciones residenciales interiores en Castilla-La Mancha, estadística elaborada a partir de las altas y bajas por cambios de residencia registradas en los padrones municipales. La evolución entre 2000 y $2009^{1}$ de esta variable revela el aumento de la población en las zonas rurales (tasas de variación positivas 2000/09) (Tabla 3) y el descenso en las urbanas (tasas de variación negativas 2000/09) (Tabla 4). Estos resultados corroboran también que son las provincias de Toledo y Guadalajara donde más se acentúa la disminución de la emigración rural y el aumento de la urbana, pero ello es fruto de la dinámica especifica de los corredores industriales de Illescas y del Henares y, por tanto, oculta, por su fuerza y dimensión, los movimientos más estandarizados en el mundo rural en estas provincias.

Tabla 3

Saldo de las variaciones residenciales municipios $<2.000$ habitantes

\begin{tabular}{|c|c|c|c|c|c|c|}
\hline- & Zonas Rurales & Albacete & Ciudad Real & Cuenca & Guadalajara & Toledo \\
\hline- & Entradas & 608 & 460 & 870 & 866 & 1355 \\
\hline $\mathbf{2 0 0 0}$ & Salidas & 986 & 742 & 1207 & 1074 & 1501 \\
\hline- & Saldo & $\mathbf{- 3 7 8}$ & $\mathbf{- 2 8 2}$ & $\mathbf{- 3 3 7}$ & $\mathbf{- 2 0 8}$ & $\mathbf{- 1 4 6}$ \\
\hline- & Entradas & 848 & 839 & 1198 & 1868 & 2176 \\
\hline $\mathbf{2 0 0 9}$ & Salidas & 1141 & 1049 & 1475 & 1693 & 2290 \\
\hline- & Saldo & $\mathbf{- 2 9 3}$ & $\mathbf{- 2 1 0}$ & $\mathbf{- 2 7 7}$ & $\mathbf{1 7 5}$ & $\mathbf{- 1 1 4}$ \\
\hline- & Tasa Variación (\%) & $\mathbf{2 2}$ & $\mathbf{2 6}$ & $\mathbf{1 8}$ & $\mathbf{1 8 4}$ & $\mathbf{2 2}$ \\
\hline
\end{tabular}

Fuente. Elaboración propia a partir de los datos del Instituto de Estadística de Castilla-La Mancha.

\footnotetext{
${ }^{1}$ Solo se utilizan los años desde 2000/09 porque son los últimos disponibles a nivel municipal para Castilla-La Mancha.
} 
Tabla 4

Saldo de las variaciones residenciales en los municipios $>10.000$ habitantes

\begin{tabular}{|c|c|c|c|c|c|c|}
\hline- & Zonas Urbanas & Albacete & Ciudad Real & Cuenca & Guadalajara & Toledo \\
\hline- & Entradas & 1975 & 2010 & 619 & 1297 & 2276 \\
\hline $\mathbf{2 0 0 0}$ & Salidas & 1271 & 1850 & 436 & 1166 & 1800 \\
\hline- & Saldo & $\mathbf{7 0 4}$ & $\mathbf{1 6 0}$ & $\mathbf{1 8 3}$ & $\mathbf{1 3 1}$ & $\mathbf{4 7 6}$ \\
\hline- & Entradas & 2953 & 3628 & 1098 & 2223 & 5559 \\
\hline $\mathbf{2 0 0 9}$ & Salidas & 2337 & 3386 & 1013 & 2665 & 5610 \\
\hline- & Saldo & $\mathbf{6 1 6}$ & $\mathbf{2 4 2}$ & $\mathbf{8 5}$ & $\mathbf{- 4 4 2}$ & $\mathbf{- 5 1}$ \\
\hline- & Tasa Variación (\%) & $\mathbf{- 1 3}$ & $\mathbf{5 1}$ & $\mathbf{- 5 4}$ & $\mathbf{- 4 3 7}$ & $\mathbf{- 1 1 1}$ \\
\hline
\end{tabular}

Fuente. Elaboración propia con datos Instituto de Estadística de Castilla-La Mancha.

En definitiva, en Castilla-La Mancha cada vez pierde más fuerza la visión de una sociedad rural que se despuebla de forma generalizada; de hecho, se encuentra actualmente en una fase de recuperación poblacional. La salida rural global ha perdido intensidad y se pone de manifiesto que el tradicional proceso de pérdida rural en los núcleos mas pequeños está siendo compensado por otro proceso inverso de emigración urbana hacia el ámbito rural (corredores) y, sobre todo, por el predominio de movimientos rural-rural en una dinámica centro-periferia.

\section{ANÁLISIS DE LA DINÁMICA DE LA POBLACIÓN RURAL: UN MODELO HEDÓNICO}

Entre las razones de este punto de inflexión en la dinámica poblacional, Frey $(1987,1989)$ apunta la periodicidad, la reestructuración regional y la descentralización. Esta última es la que ha tenido mayor acogida. Desde esta perspectiva, el nuevo espacio productivo está determinado por las preferencias de localización residencial de trabajadores y consumidores. Otros investigadores señalan las desigualdades entre áreas geográficas como la principal causa, pero impulsada por los criterios individuales de los agentes. Las personas se trasladan hacia lugares donde esperan mejorar sus condiciones de vida. Se responde, así, a los principios de racionalidad maximizadora: se busca maximizar beneficios reales, descontados los costos asociados al traslado. Por otro lado, Pérez $(1966,1971)$ habla del contraste entre mundos y situaciones; los menores ingresos de la actividad agrícola frente a los ingresos de las clases urbanas, las dificultades de acceso al consumo por la penuria en que se encontraban las redes de distribución del medio rural, el menor acceso a los servicios de información, sanitarios, de ocio y, principalmente, de enseñanza, constituyen los elementos perceptibles de una situación dispar. 
En conclusión, el éxodo rural ha estado ligado a la actividad económica, pero esta perspectiva no logra explicar todos los desplazamientos, como ya lo señalaba Pérez $(1966,1971)$. Por eso, con el propósito de interpretar y describir los motivos de los flujos migratorios entre los municipios, en este epígrafe se plantean unos modelos que, agrupando los municipios de Castilla-La Mancha, estudian el comportamiento de su población. Existen diferentes posibilidades de tomar la agrupación de municipios. Particularmente interesantes, resultan los algoritmos que aplican Pacheco y Valencia (2005) para clasificar los municipios de Castilla León siguiendo criterios económicos. Pero, para este estudio, nosotros optamos por hacer coincidir la referencia territorial con la de los Grupos de Acción Local, derivados de la aplicación de la Iniciativa Comunitaria LEADER y del Programa Operativo PRODER, en sus distintas fases, y que fueron acordados en el Plan de Zonificación aprobado por las Cortes de Castilla-La Mancha en el año 2000, puesto que es una clasificación que concentra a municipios con ciertas similitudes geográficas, sociales, históricas, económicas y culturales, y que han puesto en marcha programas de desarrollo rural de forma mancomunada.

Para realizar este análisis aplicamos la Teoría de los Modelos Hedónicos, ya que los territorios son similares a cualquier bien al que se le aplique esta teoría; son heterogéneos y divisibles, es decir, se caracterizan por una serie de atributos individuales, cada uno de los cuales contribuiría a concentrar la población. El objetivo final es obtener Modelos Hedónicos Poblacionales que demuestren la adecuación de la hipótesis previa planteada en el sentido que los aspectos económicos no son la razón principal de los desplazamientos de la población (que procede, básicamente, de la misma periferia comarcal), sino otros factores de índole social, como la oferta de servicios públicos (educación, sanidad, ocio, tecnologías de la información y comunicación) cuya trascendencia es admitida y que, como señala García-Arias (2004), son "el corpus doctrinal de la Economía". En nuestra investigación actúan como fuerzas centrípetas para la conformación de distritos rurales, en los que la terciarización en servicios productivos (de mercado) se produce a posteriori, generando la otra fuerza centrípeta de un distrito rural: las oportunidades de empleo, de inversión, y el avance en los flujos de información y el proceso de modernización económica. Ambas fuerzas centrípetas se superponen a las que puedan existir centrífugas, conformando una concentración de población y de tejido económico en lo que puede ser un centro rural a costa de la periferia de los núcleos próximos más pequeños y desfavorecidos. De modo intuitivo, Juste y Fernández (2005) ya afirman que, "en general, los mayores niveles de diversificación productiva e industrial corresponden a los municipios, cabeceras de comarca de áreas rurales, con un nivel de población medio-bajo, habiendo mantenido o aumentado ligeramente su población en los últimos 
años"; pero no establecen las relaciones de causalidad que avanzamos con nuestra investigación.

La idea subyacente para aplicar la Teoría de los Modelos Hedónicos es que, si un municipio está constituido por un conjunto de servicios, entonces su atractivo para la población deberá ser un agregado del valor individual de cada uno de ellos. Por tanto, con el objetivo de explicar la heterogeneidad inherente a un municipio, (servicios, actividad económica, localización,...), se ha considerado a éste en términos hedónicos. De tal manera, que la unidad (municipio) es conceptualizada no como bien homogéneo e indivisible, sino como una cesta de atributos individuales, cada uno de los cuales contribuye a concentrar la población. Este planteamiento responde a la Teoría de los Modelos Hedónicos.

Diferentes estudios señalan a Court (1939) como el primer investigador en acuñar el término modelo hedónico, aunque el origen de sus aplicaciones resulta más controvertido; unos señalan a Haas (1922) y otros al propio Court (1939). Lo que no hay duda es que la Teoría de los Modelos Hedónicos se ha ido perfilando con el paso del tiempo. Así, Lancaster (1966) desarrolló los fundamentos para estimar el valor de utilidad generado por las características de un bien y Rosen (1974) estableció el soporte teórico del modelo de precios hedónicos.

Simplificadamente, los modelos hedónicos establecen que los bienes están constituidos por un cierto número de atributos y que la decisión de un consumidor, en el proceso de maximización de su utilidad, al comprar un producto, viene afectada por ellos. Si extrapolamos esta idea al contexto poblacional, diremos que un modelo hedónico de población se referirá a la satisfacción que el individuo obtiene viviendo en un municipio según las características del mismo.

Dicho de otra manera, si se define $P_{i}$ como la población de un municipio $i$, ésta se puede describir por el vector $Q_{j, i}$ de los $k$ atributos de ese municipio $i$ :

$$
Q_{j, i}=\left(Q_{1 i}, Q_{2 i}, \ldots, Q_{k i}\right)
$$

A partir de aquí, se construye un modelo hedónico que explicite la relación funcional entre $P_{i}$ y sus respectivas características, $Q_{j, i}$, siendo su expresión:

$$
P_{i}=f\left(Q_{j i}, \varepsilon_{i}\right)
$$

donde $f$ es la relación funcional entre $P_{i}$ y $Q_{j, i}$, y $\varepsilon_{i}$ es la perturbación aleatoria. 
Por otro lado, la función de utilidad de residir en un municipio $i, U_{i}$, dependerá tanto de $Q_{j, i}$ como de $P_{i}$. Analíticamente, su expresión es:

$$
U_{i}=U\left(P_{i}, Q_{j, i}\right)
$$

Por tanto, un individuo obtiene su satisfacción no directamente del municipio sino de los atributos de éste. Además, decidirá habitar en un municipio $A$ frente a otro municipio $B$, si la utilidad que le proporcionen las características del primero $(A)$ son mayores que las del segundo $(B)$. En términos matemáticos sería:

$$
\frac{\partial U / \partial q_{j, A}}{\partial U / \partial A}>\frac{\partial U / \partial q_{j, B}}{\partial U / \partial B}
$$

En definitiva, los atributos $Q_{j, i}$ acercan a la población a un municipio y determinan la utilidad de vivir en él. Son fuerzas de atracción que cubren necesidades de interés comunitario, ofreciendo una mejor calidad de vida $\mathrm{y}$, como indica Dromi (2004), puede tratarse de la provisión de agua, luz, gas, teléfonos, educación, salud, transporte,.... En el contexto de Castilla-La Mancha, la provisión de luz, agua o teléfono son servicios más básicos y más universales, por lo que serían fuerzas de atracción, los servicios mínimos de sanidad $\left(Q_{\text {Sanidad }, i}\right)$, educación $\left(Q_{\text {Educación, } i}\right)$, tecnologías de la información y comunicación $\left(Q_{T I C, i}\right)$ y ocio $\left(Q_{O c i o, i}\right)$ porque el resto estarían, en general, cubiertas, y quedaría por contrastar la influencia de cierto nivel de actividad económica $\left(Q_{\text {Economia }, i}\right)$.

Teniendo en cuenta cada $Q_{j, i}$ y sustituyéndolos en la ecuación (2), $P_{i}=f\left(Q_{j i}, \varepsilon_{i}\right)$, se obtiene:

$$
P_{i}=f\left(Q_{\text {Sanidad }}, Q_{\text {Educación }}, Q_{\text {TIC }}, Q_{\text {Ocio, }}, Q_{\text {Economia }}, \varepsilon_{i}\right)
$$

en donde, se establece como condición que a medida que aumenten los servicios de un municipio, $Q_{j, i}$, su población, $P_{i}$, debiera aumentar. Matemáticamente, es la derivada parcial de la función $P_{i}=f\left(Q_{j i}, \varepsilon_{i}\right)$, con respecto a cada uno de sus atributos:

$$
\frac{\partial P_{i}}{\partial Q_{j, i}}>0
$$

o lo que es lo mismo, si consideramos la ecuación (5), las derivadas parciales para cada uno de los atributos serían: 


$$
\frac{\partial P_{i}}{\partial Q_{\text {Sanidad }}}>0, \frac{\partial P_{i}}{\partial Q_{E \text { ducación }}}>0, \frac{\partial P_{i}}{\partial Q_{T I C}}>0, \frac{\partial P_{i}}{\partial Q_{\text {Ocio }}}>0, \frac{\partial P_{i}}{\partial Q_{E \text { conomia }}}>0
$$

Por otro lado, la ecuación $P_{i}=f\left(Q_{j i}, \varepsilon_{i}\right)$, presenta aspectos no completamente resueltos, como su forma funcional $f$. La forma funcional $f$ no necesariamente corresponde a una expresión lineal, debido a que la relación entre la población y las variables explicativas suele no serlo. Es decir, a medida que aumenta la cantidad de un atributo, la magnitud del impacto sobre la población no se calcula como una razón constante. Verdaderamente, la experiencia empírica demuestra que la relación entre la variable endógena $\left(P_{i}\right)$ y las variables exógenas $\left(Q_{j, i}\right)$ tiende a adoptar formas funcionales logarítmicas. De hecho, los primeros estudiosos ensayaron diversas funciones, logarítmica, semilogarítmica, doble-logarítmica, seleccionando a éstas frente a las lineales.

Investigaciones más recientes proponen otras técnicas como la que desarrollamos en este estudio, la técnica de Box-Cox, que permite determinar la forma funcional $f$, a partir de un valor $\lambda$ que transforma a la ecuación $P_{i}=f\left(Q_{j i}, \varepsilon_{i}\right)$, en la siguiente expresión general:

$$
P_{i}^{\lambda}=\beta_{0}+\sum_{j=1}^{k} \beta_{j} Q_{j i}^{\lambda}+\varepsilon_{i}
$$

en la que $\lambda$ representa el coeficiente de Box-Cox y está comprendido $-1 \leq \lambda \leq 1$

Expresado de otra manera, sustituyendo $\sum_{j=1}^{k} \beta_{j} Q_{j i}^{\lambda}$ por los atributos determinados $\left(Q_{\text {Sanidad }, i}\right),\left(Q_{\text {Educación }, i}\right),\left(Q_{T I C, i}\right),\left(Q_{\text {Ocio }, i}\right)$ y $\left(Q_{\text {Economia }, i}\right)$, se tiene:

$$
P_{i}^{\lambda}=\beta_{0}+\beta_{1} Q_{\text {Sanidad }, i}^{\lambda}+\beta_{2} Q_{\text {Educación }}^{\lambda}+\beta_{3} Q_{\text {TIC }}^{\lambda}+\beta_{4} Q_{\text {Ocio }}^{\lambda}+\beta_{5} Q_{\text {Economia }}^{\lambda}+\varepsilon_{i}
$$

La ecuación (9) adopta diferentes formas funcionales dependiendo del valor de $\lambda$; si $\lambda=1, f$ adopta la forma lineal; si $\lambda=0$, la logarítmica. Empíricamente, en diferentes estudios, como hemos señalado anteriormente, se descarta la hipótesis de relación lineal, encontrándose valores de $\lambda$ cercanos a cero, lo que lleva a la conclusión de que la forma funcional $f$ tiende a ser muy aproximada a la logarítmica.

En nuestro caso, además, las variables exógenas son números binarios, es decir, toman valores 0 y 1 , por tanto, no es necesario efectuar la transformación 
en ellas, reduciéndose nuestra tarea a averiguar el valor de $\lambda$ que transforma a $P_{i}^{\lambda}$, de la siguiente manera:

$$
P_{j}^{*}(\lambda)=\left\{\begin{array}{lr}
P_{j}^{\lambda} & -1 \leq \lambda \leq 1 \\
\ln P_{j} & \lambda=0
\end{array} \text { y } \lambda \neq 0\right.
$$

expresándose el modelo hedónico objeto de estudio de esta forma:

$$
P_{i}^{\lambda}=\beta_{0}+\beta_{1} Q_{\text {Sanidad }, i}+\beta_{2} Q_{\text {Educación }}+\beta_{3} Q_{\text {TIC }}+\beta_{4} Q_{\text {Ocio }}+\beta_{5} Q_{\text {Economia }}+\varepsilon_{i}
$$

donde $\varepsilon_{i}$ es la perturbación aleatoria que sigue una distribución normal de media cero y varianza constante $\varepsilon_{i} \approx N\left(0, \sigma_{\varepsilon}^{2} I_{n}\right)$

La ecuación (11), independientemente del valor de $\lambda$, la estimaremos por el Método de los Mínimos Cuadrados Ordinarios, procedimiento conocido que plantea utilizar, como estimación de los parámetros, aquella combinación de $\left(\beta_{1}, \beta_{2}, \beta_{3}, \beta_{4}, \beta_{5}\right)$ que minimice la suma de los errores al cuadrado, $\sum_{i=1}^{n}\left(e_{i}\right)^{2}$, es decir, la suma de las diferencias al cuadrado entre el valor real y estimado de $P_{i}^{\lambda}$, que el modelo cometerá para las $n$ observaciones disponibles:

$$
\min \sum_{i=1}^{n}\left(e_{i}\right)^{2}=\min \sum_{i=1}^{n}\left(P_{i}^{\lambda}-\hat{P}_{i}^{\lambda}\right)^{2}
$$

Así, aplicando Mínimos Cuadrados Ordinarios a la ecuación (11) resultará:

$$
\hat{P}_{i}^{\lambda}=\beta_{0}+\hat{\beta}_{1} Q_{\text {Sanidad }, i}+\hat{\beta}_{2} Q_{\text {Educación }}+\hat{\beta}_{3} Q_{T I C}+\hat{\beta}_{4} Q_{\text {Ocio }}+\hat{\beta}_{5} Q_{\text {Economia }}
$$

estimación que someteremos a evaluación, con los diferentes contrastes sobre estructura y perturbación aleatoria, hasta que la validemos estadística y econométricamente y podamos hacer interpretaciones económicas.

En definitiva, la metodología planteada consiste en construir un modelo econométrico que explicite la relación funcional entre la población de un municipio $P_{i}$ y sus respectivas características $Q_{j, i}$, dotarlo de información estadística, estimarlo y validarlo, procesando luego los resultados para valorar cada atributo. 


\section{METODOLOGÍA HEDÓNICA DE DETERMINACIÓN DE UN DISTRITO RURAL}

El objetivo de este trabajo es determinar de entre los factores, ya presentados, sanidad $\left(Q_{\text {Sanidad }, i}\right)$, educación $\left(Q_{\text {Educación }, i}\right)$, ocio $\left(Q_{\text {Ocio }, i}\right)$, tecnologías de la información y comunicación $\left(Q_{T I C, i}\right)$ y nivel de actividad económica $\left(Q_{\text {Economia, } i}\right)$, los más influyentes en los movimientos poblacionales entre los municipios de Castilla-La Mancha $\left(P_{i}\right)$ que pertenecen al mismo Grupo de Acción Local (GAL), es decir, a un conjunto de poblaciones que comparten ciertas características. En esta región, encontramos a 31 GAL, distribuidos como aparecen en la Tabla 5:

Tabla 5

Distribución provincial de los Grupos de Acción Local que operan en Castilla-La Mancha

\begin{tabular}{|c|c|}
\hline Provincia & Grupos de Acción Local (GAL) \\
\hline Albacete & SIERRA SEGURA \\
\hline Albacete & MANCHUELA \\
\hline Albacete & SACAM \\
\hline Albacete & MANCHA JUCAR - CENTRO \\
\hline Albacete & MONTE IBERICO \\
\hline Albacete & HELLIN \\
\hline Ciudad Real & ALTO GUADIANA - MANCHA \\
\hline Ciudad Real & ALCUDIA \\
\hline Ciudad Real & TIERRAS DE LIBERTAD \\
\hline Ciudad Real & MONTESUR \\
\hline Ciudad Real & CALATRAVA \\
\hline Ciudad Real & MONTES NORTE \\
\hline Ciudad Real & PROMANCHA \\
\hline Ciudad Real & CABAÑEROS \\
\hline Cuenca & PRODESE \\
\hline Cuenca & ADIMAN \\
\hline Cuenca & ADESIMAN \\
\hline Cuenca & ALCARRIA CONQUENSE \\
\hline Cuenca & \\
\hline & ZANCARA \\
\hline & \\
\hline
\end{tabular}


Tabla 5 (continuación)

Distribución provincial de los Grupos de Acción Local que operan en Castilla-La Mancha

\begin{tabular}{|c|c|}
\hline Provincia & Grupos de Acción Local (GAL) \\
\hline Guadalajara & MOLINA DE ARAGON \\
\hline Guadalajara & ADEL \\
\hline Guadalajara & FADETA \\
\hline Guadalajara & ADAC \\
\hline Guadalajara & CORREDOR DE HENARES \\
\hline Toledo & IPETA \\
\hline Toledo & QUIJOTE \\
\hline Toledo & DULCINEA \\
\hline Toledo & CASTILLOS MEDIO TAJO \\
\hline Toledo & ADECOR \\
\hline Toledo & MONTES DE TOLEDO \\
\hline Toledo & LA SAGRA Y CORREDOR DE TOLEDO \\
\hline
\end{tabular}

Fuente: Elaboración propia.

De esta manera, se plantean 31 modelos hedónicos poblacionales como el siguiente:

$$
P_{i}^{\lambda}=\beta_{0}+\beta_{1} Q_{\text {Sanidad }, i}+\beta_{2} Q_{\text {Educación }}+\beta_{3} Q_{T I C}+\beta_{4} Q_{\text {Ocio }}+\beta_{5} Q_{\text {Economia }}+\varepsilon_{i}
$$

donde $\varepsilon_{i} \approx N\left(0, \sigma_{\varepsilon}^{2} I_{n}\right)$ es la perturbación aleatoria que sigue una distribución normal.

La ecuación (11) requiere de información estadística para ser estimada, información que se ha conseguido de diversas fuentes y que nos ha permitido conformar una base de datos de corte transversal con los 919 municipios de la región para $2008^{2}$. Las variables que se utilizan, su significado y su origen, se muestran a continuación.

La variable $\left(P_{i}\right)$ representa la población de un municipio $i$. La fuente estadística es el Instituto de Estadística de Castilla-La Mancha. Esta variable es cuantitativa, pero, a partir de ahora, las variables que presentamos son cualitativas, en concreto proxies, que nos aproximan a las variables objeto de

\footnotetext{
${ }^{2}$ Se ha seleccionado este año porque es el más reciente para el que hemos conseguido una base de datos más completa.
} 
análisis. Toman el valor "l" cuando se produce la característica mencionada y " 0 " si no se produce.

La primera variable cualitativa que se considera es una proxy a un índice económico oportunidades de empleo en el medio $\operatorname{rural}\left(Q_{E c o n o m i a, i}\right)$ para medir la actividad económica y laboral de un municipio. Se plantea como el cociente entre los contratos indefinidos $(\mathrm{CI})$ y la población potencialmente activa $(P P A)$ en el municipio $i$. Analíticamente, se formula como:

$$
Q_{\text {Economia }, i}=\frac{C I_{i}}{P P A_{i}}=\left\{\begin{array}{l}
1 \\
0
\end{array}\right\}
$$

que toma valor 1 cuando los contratos indefinidos per cápita activa de $i$ superen la media de contratos indefinidos per cápita activa del GAL. Analíticamente, su expresión es:

$$
Q_{\text {Economia }, i}=\left\{\begin{array}{l}
\text { si } \frac{C I_{i}}{P P A_{i}} \geq \frac{\sum_{i=1}^{n}\left(\frac{C I_{i}}{P P A_{i}}\right)}{n} \\
0 \text { si } \frac{C I_{i}}{P P A_{i}}<\frac{\sum_{i=1}^{n}\left(\frac{C I_{i}}{P P A_{i}}\right)}{n}
\end{array}\right.
$$

La fuente estadística de los contratos indefinidos es el Servicio Público de Empleo Estatal y de la población potencialmente activa el Instituto de Estadística de Castilla-La Mancha.

Otra variable cualitativa es la que intenta aproximarnos a la calidad sanitaria de un municipio $\left(Q_{\text {Sanidad }, i}\right)$, medida por la existencia de centros de atención sanitaria. La clasificación que realiza el Servicio de Salud de Castilla-La Mancha de centros de salud distingue entre: consultorios, centros de atención primaria y hospitales. En general, un individuo se siente más tranquilo si en el lugar donde reside existe, al menos, un centro sanitario activo las 24 horas. Por ello, hemos seleccionado, de entre todos los centros de salud, los de atención primaria. Esta variable toma valores 1 y $0, Q_{\text {Sanidad }, i}=\left\{\begin{array}{l}1 \\ 0\end{array}\right\}, 1$ sí el municipio dispone de ese tipo de centros y 0 en caso contrario. La fuente estadística utilizada ha sido el Servicio de Salud de Castilla-La Mancha.

Los centros educativos son otra fuerza tractora de la población porque es en éstos donde los niños/jóvenes complementan sus conocimientos y su base moral y social. Una posible clasificación de centros educativos es la que diferencia 
entre: colegios, institutos y universidad. Pero en este estudio ni los colegios ni la universidad son determinantes; los primeros, porque se hallan en todos los municipios; y, los segundos, porque sólo se encuentran en capitales de provincia, que están excluidas de los GAL. Por lo tanto, son los institutos los únicos que nos sirven de indicadores. De este modo, la variable $Q_{\text {Educación,i }}$ toma valor 1 si el municipio dispone de al menos un instituto y valor 0 en caso de no hallarse ninguno, $Q_{\text {Educación }, i}=\left\{\begin{array}{l}1 \\ 0\end{array}\right\}$. La fuente estadística de donde se ha obtenido esta información es la Junta de Comunidades de Castilla-La Mancha.

Por otro lado, en la actualidad son las tecnologías de la información y la comunicación, y particularmente el acceso a internet, condicionantes estructurales para la dinámica social y la económica. A partir de la base de datos de la Junta de Comunidades de Castilla-La Mancha obtenemos los habitantes con línea de internet ADSL $\left(P_{A D S L, i}\right)$, y a partir de aquí planteamos una proxy $\left(Q_{T I C, i}\right)$ como el cociente entre los habitantes con línea de internet ADSL en un municipio $\left(P_{A D S L, i}\right)$ y la población total de ese municipio $\left(P_{i}\right)$ :

$$
Q_{T I C, i}=\frac{P_{A D S L, i}}{P_{i}}=\left\{\begin{array}{l}
1 \\
0
\end{array}\right\}
$$

la variable tomará el valor 1 cuando el cociente sea mayor o igual a 0,5 , es decir, cuando la mitad o más de la población de un municipio tenga acceso a internet y tomará el valor 0 cuando no llegue a la mitad de la población la que tenga acceso a internet.

$$
Q_{T I C, i}=\left\{\begin{array}{lll}
1 & \text { si } & \frac{P_{A D S L, i}}{P_{i}} \geq 0,5 \\
0 & \text { si } \frac{P_{A D S L, i}}{P_{i}}<0,5
\end{array}\right.
$$

Por último, otra característica que hace atractivo a un municipio son los centros de ocio $\left(Q_{\text {Ocio }, i}\right)$. Hemos escogido como centros de ocio cines y teatros. La variable toma el valor 1 si el municipio dispone de un centro de ocio (teatro o cine) y 0 cuando no disponga de ellos. Los datos correspondientes a los cines se han obtenido del Ministerio de Cultura y los de teatros de la Junta de Comunidades de Castilla-La Mancha.

Con todas esas variables, podemos estimar el modelo especificado en la ecuación [11] con el paquete econométrico Eviews. 


\section{RESULTADOS}

Como ya hemos señalado en el epígrafe anterior, la primera tarea es averiguar el valor de $\lambda$ que transforma a $P$ en nuestra ecuación (11). Se han considerado los cinco valores de $\lambda$ más frecuentes que nos llevan a estudiar las ecuaciones de la Tabla 6:

\section{Tabla 6}

Valores $\lambda$ más habituales y forma funcional asociada

\begin{tabular}{|c|c|}
\hline $\begin{array}{c}\text { Valor } \\
\lambda\end{array}$ & $\begin{array}{c}\text { Ecuación } \\
\text { (forma funcional) }\end{array}$ \\
\hline$\lambda=1$ & $P_{i}=\beta_{0}+\beta_{1} Q_{\text {Sanidad }, i}+\beta_{2} Q_{\text {Educación }}+\beta_{3} Q_{T I C}+\beta_{4} Q_{\text {Ocio }}+\beta_{5} Q_{\text {Economia }}+\varepsilon_{i}$ \\
\hline$\lambda=0,5$ & $\begin{aligned} \sqrt{P_{i}}=\beta_{0}+\beta_{1} Q_{\text {Sanidad }, i}+ & \beta_{2} Q_{\text {Educación }}+\beta_{3} Q_{T I C}+\beta_{4} Q_{\text {Ocio }}+\beta_{5} Q_{\text {Economia }}+\varepsilon_{i} \\
& \text { (Raíz cuadrada en endógena) }\end{aligned}$ \\
\hline$\lambda=0$ & $\begin{array}{c}\ln P_{i}=\beta_{0}+\beta_{1} Q_{\text {Sanidad }, i}+\beta_{2} Q_{\text {Educación }}+\beta_{3} Q_{T I C}+\beta_{4} Q_{\text {Ocio }}+\beta_{5} Q_{\text {Economia }}+\varepsilon_{i} \\
\text { (Logarítmica-lineal) }\end{array}$ \\
\hline$\lambda=-0,5$ & $\begin{array}{c}1 / \sqrt{P_{i}}=\beta_{0}+\beta_{1} Q_{\text {Sanidad }, i}+\beta_{2} Q_{\text {Educación }}+\beta_{3} Q_{T I C}+\beta_{4} Q_{\text {Ocio }}+\beta_{5} Q_{\text {Economía }}+\varepsilon \\
\text { (Recíproco raíz cuadrada en endógena) }\end{array}$ \\
\hline$\lambda=-1$ & $\begin{array}{c}1 / P_{i}=\beta_{0}+\beta_{1} Q_{\text {Sanidad }, i}+\beta_{2} Q_{\text {Educación }}+\beta_{3} Q_{T I C}+\beta_{4} Q_{\text {Ocio }}+\beta_{5} Q_{\text {Economia }}+\varepsilon_{i} \\
\text { (Recíproco en endógena) }\end{array}$ \\
\hline
\end{tabular}

Fuente: Elaboración propia.

Estimadas las cinco opciones de ecuaciones, por el Método de Mínimos Cuadrados Ordinarios, para los 31 GAL, y comparando sus Sumas de Cuadrados Residuales, la mejor opción es $\lambda=0$, es decir, una función logarítmica lineal (Tabla 7), como ocurre, en general, en estudios empíricos de modelos hedónicos. 
Tabla 7

Elección de $\lambda$ según la Suma de Cuadrados Residual

\begin{tabular}{|c|c|c|c|c|c|c|}
\hline \multirow[b]{2}{*}{ Provincia } & \multirow[b]{2}{*}{$\begin{array}{c}\text { Grupos de Acción Local } \\
\text { (GAL) }\end{array}$} & \multicolumn{5}{|c|}{ Suma de Cuadrados Residual* } \\
\hline & & $\lambda=1$ & $\lambda=0,5$ & $\lambda=0$ & $\lambda=-0,5$ & $\lambda=-1$ \\
\hline Albacete & SIERRA SEGURA & 5280378 & 627,8 & 1,5 & $0^{*}$ & $0^{* *}$ \\
\hline Albacete & MANCHUELA & 6879947 & 932,8 & 5,9 & $0^{*}$ & $0^{* *}$ \\
\hline Albacete & SACAM & 4954352, & 851,25 & 5 & $0^{*}$ & $0^{* *}$ \\
\hline Albacete & MANCHA JUCAR - CENTRO & 1,41E+08 & 2388,12 & 1,3 & $0^{*}$ & $0^{* *}$ \\
\hline Albacete & MONTE IBERICO & 28078335 & 1131,35 & 0,8 & $0^{*}$ & $0^{* *}$ \\
\hline Albacete & HELLIN* & $2,64 E+08$ & 3730 & 0,9 & $0^{*}$ & $0^{* *}$ \\
\hline Ciudad Real & $\begin{array}{l}\text { ALTO GUADIANA - } \\
\text { MANCHA }\end{array}$ & 78680988 & 1739,31 & 1,3 & $0^{*}$ & $0^{* *}$ \\
\hline Ciudad Real & ALCUDIA & 5334795 & 313 & 0,6 & $0^{*}$ & $0^{\star *}$ \\
\hline Ciudad Real & TIERRAS DE LIBERTAD & 10813115 & 1382 & 4,2 & $0^{*}$ & $0^{* *}$ \\
\hline Ciudad Real & MONTESUR & 2005546, & 487,5 & 2,3 & $0^{*}$ & $0^{\star *}$ \\
\hline Ciudad Real & CALATRAVA & 22733364 & 2091 & 8 & $0^{*}$ & $0^{* *}$ \\
\hline Ciudad Real & MONTES NORTE & 8270052 & 1557 & 6,8 & $0^{*}$ & $0^{* *}$ \\
\hline Ciudad Real & PROMANCHA & $5,49 E+08$ & 6717,5 & 1,4 & $0^{*}$ & $0^{* *}$ \\
\hline Ciudad Real & CABAÑEROS & 1220378, & 199 & 0,5 & $0^{*}$ & $0^{* *}$ \\
\hline Cuenca & PRODESE & 1881666, & 1394,41 & 28 & $0^{*}$ & $0^{* *}$ \\
\hline Cuenca & ADIMAN & 28218855 & 2707,7 & 14 & $0^{*}$ & $0^{\star *}$ \\
\hline Cuenca & ADESIMAN & $1,17 E+08$ & 5752 & 27,2 & $0^{*}$ & $0^{* *}$ \\
\hline Cuenca & ALCARRIA CONQUENSE & 460371,0 & 686,7 & 25 & $0^{*}$ & $0^{* *}$ \\
\hline Cuenca & ZANCARA & 10010526 & 1269 & 9,3 & $0^{*}$ & $0^{* *}$ \\
\hline Guadalajara & MOLINA DE ARAGON & 293663,7 & 649,3 & 35 & $0^{*}$ & $0^{\star *}$ \\
\hline Guadalajara & ADEL & 14640093 & 2138,12 & 35,2 & $0^{*}$ & $0^{\star *}$ \\
\hline Guadalajara & FADETA & 9045610 & 3437 & 44,6 & $0^{*}$ & $0^{\star *}$ \\
\hline Guadalajara & ADAC & 27147815 & 2902 & 25 & $0^{*}$ & $0^{* *}$ \\
\hline Guadalajara & CORREDOR DE HENARES & & & & & \\
\hline Toledo & IPETA & $4,10 \mathrm{E}+09$ & 35164,90 & 22 & $0^{*}$ & $0^{\star *}$ \\
\hline Toledo & QUIJOTE & 20844513 & 2590 & 7,4 & $0^{*}$ & $0^{\star *}$ \\
\hline Toledo & DULCINEA & 39645646 & 1940 & 3 & $0^{*}$ & $0^{* *}$ \\
\hline Toledo & CASTILLOS MEDIO TAJO & 72508081 & 6549 & 19,8 & $0^{*}$ & $0^{* *}$ \\
\hline Toledo & ADECOR & 11435160 & 1709,6 & 5,8 & $0^{*}$ & $0^{* *}$ \\
\hline Toledo & MONTES DE TOLEDO & 38189899 & 3332 & 14,5 & $0^{*}$ & $0^{* *}$ \\
\hline Toledo & $\begin{array}{l}\text { LA SAGRA Y CORREDOR } \\
\text { DE TOLEDO }\end{array}$ & $1,62 E+08$ & 5104 & 4,2 & $0^{*}$ & $0^{\star *}$ \\
\hline
\end{tabular}

* Se escogen los modelos con menor Suma de Cuadrados Residual.

** Las últimas dos columnas, aunque las sumas obtenidas son muy próximas a cero, no se tienen en cuenta porque, en general, los coeficientes estimados son negativos para atraer a la población.

* El Corredor de Henares no lo hemos podido estimar porque todos los municipios que lo integran disponen de los servicios mínimos.

Fuente: Elaboración propia. 
Después, para comprobar si el modelo cumple las Hipótesis Clásicas, analizamos la significación individual (t-Student), conjunta (F-Snedecor), hetorocedasticidad (test de White), autocorrelación (Breusch-Godfrey), y bondad $\left(R^{2}\right)$; corregimos las contrariedades derivadas de la especificación y estimación y, de ser necesario, reestimamos los modelos. Un resumen de los resultados obtenidos se presenta en las Tablas 8 y 9 .

Analizando los resultados de la Tabla 6 se deduce que, a nivel individual, el atributo de la educación, $Q_{\text {Educación }, i}$, es el factor que más incide para explicar la dinámica de la población de Castilla-La Mancha, con presencia en el 71\% de los $G A L$; le sigue en importancia el factor sanidad $Q_{\text {Sanidad }, i}$, con una ponderación del $52 \%$, y el factor tecnologías de la información y comunicación, $Q_{T I C, i}$, con un peso del $35,5 \%$ de los $G A L$; menos significativo se muestra el atributo ocio $Q_{\text {Ocio }, i}$ con un $32 \% \mathrm{y}$, en último lugar, la economía (oportunidades de empleo) $Q_{\text {Economia }, i}$ con tan solo un $13 \%$.

Ahora bien, en el conjunto de los atributos, $Q_{j, i}$, los resultados muestran que, en general, en los $G A L$, ningún factor se presenta como único determinante en el arrastre de la población, sino la combinación de al menos dos factores, en los que el denominador común es sanidad y/o educación.

Otro aspecto a destacar es, que en las provincias de Cuenca y Guadalajara es donde más se valora el acceso a las tecnologías de la información y comunicación.

Por último, debe señalarse que el factor económico no es el detonante en los movimientos poblacionales entre municipios pertenecientes a un mismo GAL. En tan sólo 4 de los 31 GAL resulta significativo el atributo, $Q_{E c o n o m i a, i}$; en concreto en La Manchuela (Albacete), Monte Ibérico (Albacete), Cabañeros (Ciudad Real), y La Sagra (Toledo).

En definitiva, como valoración global, queda confirmada la hipótesis de que el surgimiento de actividades terciarias publicas y de servicios básicos de nomercado (educación, sanidad, nuevas tecnologías, centros de ocio,...) en el medio rural, sería la fuerza de atracción para la población, que a su vez llevaría, a posteriori, a un aumento de la actividad económica y reproducirían, en un nivel distintivo, fenómenos similares, pero de base productiva en lugar de social, extendidos formalmente en los clusters y distritos industriales, conformándose así un nuevo concepto en el desarrollo rural: el Distrito Rural. Estructura y ente que se presenta en las comarcas rurales y explica la dinámica de población y la base económica de los núcleos rurales en espacios homogéneos, decantando así en una gama de sistemas de centros-periferia rurales. 
Tabla 8

Factores $Q_{j, i}$ que influyen en la población

\begin{tabular}{|c|c|c|c|c|c|c|}
\hline \multirow[b]{2}{*}{ Provincia } & \multirow[b]{2}{*}{$\begin{array}{l}\text { Grupo Desarrollo Rural } \\
\text { (GDR) }\end{array}$} & \multicolumn{5}{|c|}{$\begin{array}{c}\text { Probabilidad asociada a la T Student Factores } \\
\text { Se consideran significativos al } 90 \%\end{array}$} \\
\hline & & ECONOMÍA & SANIDAD & EDUCACIÓN & TIC & OCIO \\
\hline Albacete & SIERRA SEGURA & _ & _ & 0,014 & _ & _ \\
\hline Albacete & MANCHUELA & 0,03 & 0,06 & 0,14 & 0,11 & 0,01 \\
\hline Albacete & SACAM & 0,25 & 0,009 & 0,04 & & 0,35 \\
\hline Albacete & MANCHA JUCAR - CENTRO & 0,64 & 0,02 & 0,10 & _ & 0,45 \\
\hline Albacete & MONTE IBERICO & 0,028 & - & 0,004 & _ & 0,03 \\
\hline Albacete & HELLIN* & _ & 0,16 & 0,14 & _ & _ \\
\hline Ciudad Real & $\begin{array}{l}\text { ALTO GUADIANA - } \\
\text { MANCHA }\end{array}$ & 0,94 & 0,010 & 0 & - & 0,79 \\
\hline Ciudad Real & ALCUDIA & 0,31 & 0,33 & 0,008 & 0,15 & 0,02 \\
\hline Ciudad Real & TIERRAS DE LIBERTAD & 0,41 & 0,08 & 0,003 & - & 0,005 \\
\hline Ciudad Real & MONTESUR & $\ldots$ & 0,033 & - & _ & - \\
\hline Ciudad Real & CALATRAVA & 0,13 & 0,014 & - & _ & 0,17 \\
\hline Ciudad Real & MONTES NORTE & - & $\ldots$ & 0,04 & _ & - \\
\hline Ciudad Real & PROMANCHA & _ & 0,0055 & - & _ & _ \\
\hline Ciudad Real & CABAÑEROS & 0,07 & - & 0,02 & _ & _ \\
\hline Cuenca & PRODESE & 0,53 & 0,0048 & 0,45 & 0,0004 & 0,37 \\
\hline Cuenca & ADIMAN & 0,85 & _ & 0,0005 & 0,0085 & 0,24 \\
\hline Cuenca & ADESIMAN & 0,83 & 0,08 & 0,005 & 0,0032 & 0,04 \\
\hline Cuenca & ALCARRIA CONQUENSE & $\ldots$ & 0,016 & & 0,0006 & 0,02 \\
\hline Cuenca & ZANCARA & _- & 0,0095 & & 0,0032 & 0,04 \\
\hline Guadalajara & MOLINA DE ARAGON & 0,67 & 0,04 & 0,03 & 0,0002 & - \\
\hline Guadalajara & ADEL & 0,41 & 0,25 & 0,0012 & 0,037 & 0,015 \\
\hline Guadalajara & FADETA & _ & 0,08 & 0 & 0,002 & \\
\hline Guadalajara & ADAC & _ & $\ldots$ & 0,02 & 0,07 & \\
\hline Guadalajara & CORREDOR DE HENARES & _ & - & _ & - & - \\
\hline Toledo & IPETA & 0,13 & - & 0,02 & _ & 0,0005 \\
\hline Toledo & QUIJOTE & _ & 0,0152 & _ & _ & _ \\
\hline Toledo & DULCINEA & 0,78 & 0,50 & 0,0013 & - & 0,43 \\
\hline Toledo & CASTILLOS MEDIO TAJO & _ & 0,17 & 0,04 & 0,09 & _ \\
\hline Toledo & ADECOR & _ & 0,15 & 0,0041 & 0,02 & - \\
\hline Toledo & MONTES DE TOLEDO & 0,43 & 0,20 & 0,0084 & - & 0,0003 \\
\hline Toledo & $\begin{array}{c}\text { LA SAGRA Y CORREDOR } \\
\text { DE TOLEDO }\end{array}$ & 0,0018 & - & 0,0003 & - & 0,19 \\
\hline
\end{tabular}

* Las casillas sombreadas son las significativas.

* No todas las casillas aparecen con valor de probabilidad porque ha sido necesario rehacer el modelo, omitiendo variables que no resultaban significativas.

* El Corredor de Henares no lo hemos podido estimar porque todos los municipios que lo integran disponen de los servicios mínimos.

Fuente: Elaboración propia. 
Tabla 9

Resumen de hipótesis clásicas

\begin{tabular}{|c|c|c|c|c|c|}
\hline Provincia & $\begin{array}{l}\text { Grupos de Acción } \\
\text { Local (GAL) }\end{array}$ & $\begin{array}{l}\text { Significatividad } \\
\text { Conjunta } \\
\text { F Snedecor } \\
(p<0,10)\end{array}$ & $\begin{array}{c}\text { Ausencia } \\
\text { Heterocedasticidad } \\
\text { Contraste de White } \\
\text { para sección cruzada } \\
(p>0,10)\end{array}$ & $\begin{array}{c}\text { Ausencia } \\
\text { Autocorrelacion } \\
\text { Breusch-Godfrey } \\
(p>0,10)\end{array}$ & $R 2$ \\
\hline Albacete & SIERRA SEGURA & 0,0033 & 0,56 & 0,25 & $60 \%$ \\
\hline Albacete & MANCHUELA & 0 & 0,57 & 0,28 & $80 \%$ \\
\hline Albacete & SACAM & 0 & 0,45 & 0,72 & $73 \%$ \\
\hline Albacete & $\begin{array}{l}\text { MANCHA JUCAR - } \\
\text { CENTRO }\end{array}$ & 0,01 & - & 0,99 & $94 \%$ \\
\hline Albacete & MONTE IBERICO & 0 & 0,3 & 0,48 & $90 \%$ \\
\hline Albacete & HELLIN & 0,13 & - & 0,08 & $86 \%$ \\
\hline Ciudad Real & $\begin{array}{l}\text { ALTO GUADIANA - } \\
\text { MANCHA }\end{array}$ & 0 & 0,08 & 0,08 & $94 \%$ \\
\hline Ciudad Real & ALCUDIA & 0 & - & 0,84 & $94 \%$ \\
\hline Ciudad Real & TIERRAS LIBERTAD & 0 & 0,20 & 0,43 & $80 \%$ \\
\hline Ciudad Real & MONTESUR & 0,033 & 0,90 & 0,97 & $55 \%$ \\
\hline Ciudad Real & CALATRAVA & 0,006 & 0,23 & 0,49 & $73 \%$ \\
\hline Ciudad Real & MONTES NORTE & 0,008 & 0,41 & 0,26 & $52 \%$ \\
\hline Ciudad Real & PROMANCHA & 0,0055 & 0,23 & 0,13 & $81 \%$ \\
\hline Ciudad Real & CABAÑEROS & 0,01 & 0,18 & 0,21 & $82 \%$ \\
\hline Cuenca & PRODESE & 0 & 0,85 & 0,15 & $52 \%$ \\
\hline Cuenca & ADIMAN & 0 & 0,75 & 0,77 & $81 \%$ \\
\hline Cuenca & ADESIMAN & 0 & 0,94 & 0,41 & $62 \%$ \\
\hline Cuenca & $\begin{array}{l}\text { ALCARRIA } \\
\text { CONQUENSE }\end{array}$ & 0 & 0,47 & 0,22 & $56 \%$ \\
\hline Cuenca & ZANCARA & 0 & 0,45 & 0,41 & $63 \%$ \\
\hline Guadalajara & MOLINA DE ARAGON & 0 & 0,91 & 0,55 & $50 \%$ \\
\hline Guadalajara & ADEL & 0 & 0,95 & 0,89 & $58 \%$ \\
\hline Guadalajara & FADETA & 0 & 0,56 & 0,77 & $60 \%$ \\
\hline Guadalajara & ADAC & 0 & 0,36 & 0,93 & $75 \%$ \\
\hline Guadalajara & $\begin{array}{l}\text { CORREDOR DE } \\
\text { HENARES }\end{array}$ & - & - & - & - \\
\hline Toledo & IPETA & 0 & 0,16 & 0,54 & $67 \%$ \\
\hline Toledo & QUIJOTE & 0,015 & 0,23 & 0,20 & $35 \%$ \\
\hline Toledo & DULCINEA & 0,0014 & 0,55 & 0,16 & $78 \%$ \\
\hline Toledo & $\begin{array}{c}\text { CASTILLOS MEDIO } \\
\text { TAJO }\end{array}$ & 0 & 0 & 0,34 & $65 \%$ \\
\hline Toledo & ADECOR & 0 & 0,39 & 0,19 & $66 \%$ \\
\hline Toledo & MONTES DE TOLEDO & 0 & 0,91 & 0,02 & $71 \%$ \\
\hline Toledo & $\begin{array}{l}\text { LA SAGRA Y } \\
\text { CORREDOR } \\
\text { DE TOLEDO }\end{array}$ & 0 & 0,07 & 0,21 & $59 \%$ \\
\hline
\end{tabular}

Fuente: Elaboración propia. 


\section{CONCLUSIONES}

La dinámica demográfica en el mundo rural actual está guiada por un proceso reciente, que es más complejo y más triangular que los cambios experimentados en décadas anteriores, en las que la disminución de la población en todos los núcleos rurales era generalizada. Actualmente, los dos signos distintivos son: los movimientos rural-rural y los corredores periurbanos. El movimiento de la población se realiza de los núcleos más pequeños (periferia) a la cabecera comarcal (centro), en general. Los núcleos de población grandes e intermedios ya no solo no pierden población sino que la ganan y se conforman como centros sociales y productivos compactos y consistentes. Las razones de estos flujos no son explicados, básicamente, por una causa de índole económica, sino por la existencia de una dotación más alta de infraestructuras sociales que ejercen de modelo gravitacional como una economía de escala social.

Por tanto, aparecen los núcleos rurales mas grandes como concentradores de la población absorbida de los núcleos periféricos, conformando un 'distrito social' frente al tradicional distrito industrial de tradición marshalliana, y que nosotros denominamos como Distrito Rural, que se convierte, así, en un nuevo concepto para el desarrollo territorial. Esta génesis de un sistema centroperiferia intrarural viene derivada, directamente, de la presencia de economías de escala sociales, presente en la dotación de infraestructuras básicas en estas poblaciones, que adquieren esas fuerzas centrípetas (en sentido de la geografía económica de Krugman). Desde esta perspectiva, los ámbitos rurales centrales se han revalorizado y son las ventajas residenciales y sociales que éstos ofrecen las que motivan a los individuos a residir en unos frente a otros núcleos periféricos, a lo que se añade que la terciarización de servicios de mercado rural es subsecuente a la dinámica demográfica, tal y como se avanza con los Modelos Hedónicos Poblacionales, que se han adaptado, a estos efectos, en el presente artículo, como novedad metodológica.

Aunque se ha utilizado Castilla-La Mancha como estudio de caso, la investigación se está ampliando a la totalidad del territorio de España, y se está confirmando la validez de las premisas y resultados alcanzados para el caso manchego. El tradicional fenómeno de concentración de población en los núcleos urbanos empezó a cambiar en 1980, produciéndose un proceso de recuperación demográfica de las zonas rurales, sobre todo en Toledo y Guadalajara. García (2003) señala que el desarrollo de los mecanismos de servicios, información y de innovación tecnológica y, por ende, su impacto sobre el mercado de trabajo, han generado nuevas formas de poblamiento en las zonas rurales. Esta idea se confirma para Castilla-La Mancha, según los resultados que se desprenden de los Modelos Hedónicos Poblacionales planteados para el periodo de la última década. En éstos se concluye que la actividad económica ya no es la principal razón de los movimientos migratorios, 
sino la disposición de servicios sociales básicos, sobre todo sanitarios y de educación.

Una vez configurada la concentración de población, se genera una osmosis a la terciarización de sesgo privada, complementaria a la pública, que a su vez conforma nuevas oportunidades y ejerce de fuerza centrípeta. Valoremos que una importante ventaja de la especialización terciaria (sin paso previo por la fase de industrialización) es que proporciona diversas fuentes de empleo para la mayor parte de la población activa, incluso en las zonas rurales. De hecho, en Castilla-La Mancha, región eminentemente rural, el 59\% de los puestos de trabajo se crean en este sector que supone un $61 \%$ del PIB de la región. Por otro lado, las desigualdades geográficas del sector terciario de no mercado, que generan desequilibrios espaciales en cuanto al nivel de vida (acceso a la educación y a la asistencia médico-sanitaria, posibilidades de acceder a los servicios recreativos y culturales,...), configuran restricciones sistémicas que son restricciones a priori, pero también a posteriori; por tanto, son origen y también mecanismos de causalidad acumulativa del proceso.

La conclusión final también debe referirse a las implicaciones de nuestra constatación para la política de desarrollo rural: valorar si es un modelo a aceptar o a combatir. En el sistema centro-periferia rural, la población puede mantenerse en el medio rural pero hay una restructuración y resituación espacial con un desequilibrio para las zonas rurales más periféricas y desfavorecidas. Si se acepta, el mecanismo a fortalecer es claro: para atraer a la población, impulsar la economía y acelerar los procesos de cambio y homogeneización en lo que se refiere al nivel de vida entre zonas rurales y urbanas; si se debe combatir también las implicaciones y relaciones de causalidad son patentes: la dotación de infraestructuras sociales y el coste a asumir, con el correspondiente análisis coste-beneficio, deben ser acometidos. Esta es una de las nuevas líneas de trabajo que estamos desarrollando, pero lo que es indudable es que la política de desarrollo territorial, y dentro de ella, la política de desarrollo rural, tiene un nuevo reto y una necesidad ineludible de redefinirse y reorientarse, pues la presencia de los Distritos Rurales es una realidad que debe configurar un eje central en la valoración de nuevos objetivos e instrumentos de esta política pública.

\section{REFERENCIAS BIBLIOGRÁFICAS}

ALLENDE, A. (1991). "Crisis metropolitana y Enterprises zones. Una nueva experiencia en la política urbana”. Ciudad y Territorio, 55, pp.37-50. 
ARROYO, M. (2001). "La contraurbanización: un debate metodológico y conceptual sobre la dinámica de las áreas metropolitanas". Artículo. http://www.ub.edu/geocrit/sn-97.htm. [Último acceso: Marzo de 2011].

BELIL, M.I.C. (1990). "Industrialización y espacio urbano". Documents d'analisis Geografica, 17, pp.121-131.

BENITO, P. (1991). "Descentralización productiva y nuevas formas de agrupación Industrial: los corredores industriales de Asturias". Ciudad y Territorio, 90, pp.365-371.

BERNABÉ, J.M. y ALBERTOS, J.M. (1986). "Migraciones interiores en España". Cuadernos de Geografía, 39-40, pp.175-202.

CAMARERO, L.A. (1993). Del éxodo rural y del éxodo urbano. Ocaso y renacimiento de los asentamientos rurales en España. Madrid: Ministerio de Agricultura, Pesca y Alimentación.

COURT, A.T. (1939). The Dynamics of Automobile Demand. New York: General Motors.

ENTRENA, F. La desterritorialización de las comunidades locales rurales y su creciente consideración como unidades de desarrollo. Artículo. http://cederul.unizar.es/revista/num03/pag03.htm. [Último acceso: Marzo de 2011].

FERRÁS, C. (2007). "El enigma de la contraurbanización. Fenómeno empírico y concepto caótico". Documento de trabajo. http://redalyc.uaemex.mx/pdf/196/19609801.pdf. [Último acceso: Marzo de 2011].

FREY, W. (1987). "Migration and depopulation of the metrópolis: regional restructuring or rural renaissance?". American Sociological Review, 52, pp.240-257.

FREY, W. (1989). "United Estates: Counterurbanization and metropolis depopulation" en Champion, A.G. (ed): Counterurbanization. The changing pace and nature of population deconcentration. Londres: Edward Arnold.

GARCÍA, B. (1997). La sociedad rural ante el siglo XXI. Madrid: Ministerio de Agricultura, Pesca y Alimentación.

GARCíA, B. (2003): Sociedad rural y desarrollo. Madrid: Ministerio de Agricultura, Pesca y Alimentación.

GARCÍA-ARIAS, J. (2004). "Un nuevo marco de análisis para los bienes públicos: la Teoría de los Bienes Públicos Globales". Revista de Estudios de Economía Aplicada, 22(2), pp. 187-212.

HASS, G.C. (1922). "Sales prices as a basis for farm land appraisal". Technical Bulletin of the University of Minnesota, Agricultural Experiment Station, 9.

JUSTE, J.J. y FERNÁNDEZ. J.E. (2005). "Sistemas productivos locales en Castilla y León: Identificación y rasgos básicos". Revista de Estudios de Economía Aplicada, 23(2), pp. 423-450. 
KRUGMAN, P. (1991). "Increasing Returns and Economic Geography". Journal of Political Economy, 99 (3), pp. 183-199.

LANCASTER, K. J. (1966). "A new approach to consumer theory". Journal of Political Economy, 74, pp.132-57.

OGANDO, O.; PEDROSA, R. y FERNÁNDEZ, J.E. (1989). "Análisis territorial de la inversión industrial en las regiones españolas". Estudios Territoriales, 37, pp.79-94.

PACHECO, J. y VALENCIA. O. (2005). "Análisis de nuevos métodos de clasificación. Un ejemplo ilustrativo de su uso en la agrupación de los municipios de Castilla y León". Revista de Estudios de Economía Aplicada, 23(3), pp. 711-729.

PÉREZ, V. (1966). Estructura social del campo y del éxodo rural. Madrid: Tecnos.

PÉREZ, V. (1971). Emigración y cambio social: procesos migratorios y vida rural en Castilla. Barcelona: Ariel.

ROSEN, S. (1974). "Hedonic prices and implicit markets: product differentiation in pure competition". Journal of Political Economy, 83, pp.34- 55. 